Artículo

\title{
Evaluación de la sustentabilidad del cultivo de maíz en Villaflores y La Trinitaria, Chiapas
}

\author{
Salvador González Flores ${ }^{1}$ \\ Lenin G. Guajardo Hernández ${ }^{1 \S}$ \\ S. Xochilt Almeraya-Quintero ${ }^{1}$ \\ Luz María Pérez-Hernández ${ }^{1}$ \\ Dora Ma. Sangerman-Jarquín ${ }^{2}$
}

${ }^{1}$ Colegio de Posgraduados-Posgrado en Socio Economía, Estadística e Informática-Desarrollo Rural. Carretera México-Texcoco km 36.5, Montecillo, Texcoco, Estado de México. (xalmeraya@colpos.mx; luzmaph@colpos.mx). CP. 56230. Tel. 595 9520200, ext. 1876. ${ }^{2}$ Campo Experimental Valle de MéxicoINIFAP. Carretera Los reyes-Texcoco km 13.5, Coatlinchán, Estado de México. CP. 56250. (sangerman.dora@inifap.gob.mx).

$\S^{\S}$ Autor para correspondencia: glenin@ colpos.mx.

\section{Resumen}

Hoy en día, los grandes sucesos ambientales han tenido una repercusión sobre la población en sus diferentes niveles económicos y sociales y sobre todo, en la producción agrícola. De ahí que, la sustentabilidad constituye una prioridad ante la necesidad de desarrollo que no solo involucra a los recursos naturales, sino que también se interrelaciona con aspectos económicos y sociales. El objetivo de esta investigación radica en el interés de medir la sustentabilidad del sistema de producción de maíz, a fin de determinar los niveles de sustentabilidad del sistema productivo, y formular alternativas que coadyuven al diseño del sistema sustentable que beneficien la productividad con la equidad social, la conservación de los recursos naturales y la toma de decisiones de las unidades productivas para el año 2018. Por lo que se evaluó mediante el marco de evaluación de sistemas de manejo incorporando indicadores de sustentabilidad. A partir de los resultados, se demuestra que los valores de sustentabilidad para los municipios de Villaflores y La Trinitaria, son de 3.78 y 4.81, respectivamente, predominando un nivel sustentable limitado. Se concluye, que la sustentabilidad del sistema productivo podrá mantenerse en condiciones óptimas y estables, en la medida que se realicen cambios en los beneficios económicos, autodependencia, equidad participativa en el proceso de producción y en la toma de decisiones, implementación de técnicas agroecológicas y adopción de prácticas sustentables y el manejo sostenido del cultivo de maíz para preservar y conservar el recurso natural de forma integral.

Palabras clave: agroecosistema, desarrollo sustentable, metodología MESMIS, técnicas agroecológicas.

Recibido: febrero de 2020

Aceptado: junio de 2020 


\section{Introducción}

Hoy en día, se requiere asegurar patrones de desarrollo sustentable en los sistemas de producción agrícola, promovidos como agroecosistemas sustentables desde el punto de vista ambiental y sociocultural (Astier et al., 2002). El incremento poblacional, el deterioro ambiental y el modelo de la agricultura intensiva, ha generado interés en la sociedad sobre la creación de nuevas estrategias encaminadas al desarrollo sustentable, que logren la producción de alimentos sin afectar los ecosistemas naturales (ONU, 1992).

Lo que conlleva a generar rendimientos sostenidos a largo plazo a través del uso de tecnologías y prácticas que mejoren la eficiencia del sistema de producción; y no altos rendimientos a corto plazo. Es decir, se busca la optimización del sistema mediante las interacciones de los componentes bióticos y abióticos (Altieri, 1994). Por otro lado, grandes sucesos ambientales han tenido una repercusión sobre la población en sus diferentes niveles económicos y sociales y sobre todo, en la producción agrícola. La actividad humana implica mantener contacto con el medio, lo cual conduce a la necesidad de identificar la región en que se actúa, para producir mejor y cooperar de manera más eficiente con el desarrollo de la sociedad (Bassols, 1984).

Así, la sustentabilidad en el contexto de los sistemas agrícolas considera la creación de sistemas productivos de recursos naturales, estables y adaptables, que distribuyan los costos y beneficios en forma equitativa, generando procesos autogestivos entre otros beneficiarios (Masera et al., 2000). Por su parte, Edwards et al. (1990) refiere que la agricultura sustentable no solo se refiere a una agricultura que intenta proporcionar rendimientos sostenidos a largo plazo, sino que ha promovido la necesidad de realizar ajustes en la agricultura convencional para que éste se vuelva ambiental, social, y económicamente viable y compatible.

Asimismo, se requiere cambiar los patrones de crecimiento, los hábitos de consumo, la inversión de infraestructura y tecnología que contrarresten la problemática del deterioro ambiental a fin de alcanzar la conservación y desarrollo sustentable. En este sentido, para lograr medir la sustentabilidad de los sistemas productivos, es necesario identificar y evaluar las variables sociales, económicas y ecológicas involucradas en el desarrollo, mediante procesos integradores, que generen indicadores, que sean holísticos para analizar y evaluar el estado del ambiente y las funciones ecológicas, así como el impacto y las consecuencias del desarrollo en los recursos naturales (Torres et al., 2000).

El diseño de sistemas sustentables debe estar orientada a pequeños productores de escasos recursos económicos, reducir los costos de producción y el aumento de los beneficios, tales como la productividad, la conservación de los conocimientos tradicionales relacionados con el manejo agrícola, así como el acceso a los insumos, alimentos y mercados (Astier et al., 2008). De ahí que, la sustentabilidad constituye una prioridad ante la necesidad de desarrollo que no solo involucra a los recursos naturales, sino que también se interrelaciona con aspectos económicos y sociales.

Por lo tanto, el objetivo del presente trabajo de investigación radica en el interés de medir la sustentabilidad del sistema de producción agrícola con mayor trascendencia en la región del estado de Chiapas, lo que constituye el cultivo de maíz, mediante el marco de evaluación de sistemas de manejo incorporando indicadores de sustentabilidad (MESMIS), a fin de determinar los niveles de 
sustentabilidad del sistema productivo, y formular alternativas que coadyuven al diseño del sistema sustentable, beneficien la productividad con la equidad social, la conservación de los recursos naturales y la toma de decisiones de las unidades productivas.

\section{Materiales y métodos}

El presente trabajo de investigación es de tipo mixto, dado que tiene alcance observacional, exploratorio, descriptivo, no experimental y transversal. Por otra parte, en cuanto al marco muestral, se determinó mediante la selección de productores de los municipios de Villaflores y La Trinitaria, Chiapas, utilizando el padrón de beneficiarios de Proagro Productivo del ciclo agrícola 2017.

Enseguida, se ocupó el muestreo estratificado ya que, en este tipo, la población se divide en estratos, y tiende a mayor precisión que en comparación al muestreo aleatorio simple. En este sentido, de acuerdo con Vivanco (2005) para determinar el tamaño de los estratos del muestreo, se asignaron de acuerdo con el estrato de pertenencia, atendiendo el criterio de la concentración de $80 \%$ de productores de maíz en las localidades de los municipios de Villaflores y La Trinitaria, Chiapas.

Finalmente, la estratificación, comprendió de seis y cinco localidades, para cada uno de los municipios. Respecto a la afijación utilizada para el muestreo estratificado, se determinó afijación proporcional. Según Vivanco (2005), esta afijación presenta como ventaja, que se genera una muestra autoponderada caracterizada por la misma fracción de muestreo en todos los estratos.

Posteriormente, se aplicó la siguiente fórmula estadística que representa el muestreo estratificado afijación proporcional (Vivanco, 2005).

Tamaño total de la muestra

$$
n=\frac{\Sigma_{i=1}^{l} N_{i} P_{i} Q_{i}}{N E+\frac{1}{N} \Sigma_{i=1}^{l} N_{i} P_{i} Q_{i}} \quad E=\frac{d^{2}}{Z_{1-\alpha / 2}^{2}}
$$

Tamaño de cada estrato

$$
n_{i}=n\left(\frac{N_{i}}{\Sigma_{i=1}^{I} N_{i}}\right)=n\left(\frac{N_{i}}{N}\right)=n\left(W_{i}\right)
$$

El porcentaje de confianza para esta investigación es de $95 \%$ y un error de precisión de $10 \%$. Donde: $\mathrm{N}=$ tamaño de la población es de 844 productores para el municipio de Villaflores y 992 productores correspondiente al municipio de La Trinitaria; $\mathrm{N}_{\mathrm{i}}=$ tamaño de la población del estrato; $\mathrm{P}_{\mathrm{i}}=$ proporción esperada de $20 \% ; \mathrm{Q}_{\mathrm{i}}=1-\mathrm{P}_{\mathrm{i}}(1-0.2=0.80) ; d=$ error absoluto o precisión de $10 \%$; $\mathrm{Z}_{1-\alpha / 2}=$ nivel de confianza de $95 \%$, por tanto $\mathrm{Z}_{1-\alpha / 2}=1.96 ; \mathrm{NE}=$ producto del tamaño de la población por el error de estimación; $\mathrm{n}=$ tamaño total de la muestra; $\mathrm{y} \mathrm{n}_{\mathrm{i}}=$ tamaño de cada estrato. 
Entonces, el tamaño de muestra $n$ estimado en el caso del municipio de Villaflores y La Trintiaria, fue de 57 y 58 encuestas, respectivamente que representa el total de la población objetivo de la zona de estudio. En otro tenor, se determinó el tamaño de cada estrato mediante la fórmula correspondiente sobre afijación proporcional, obteniendo la muestra para cada conglomerado.

Con respecto a la recolección de información de campo, se realizó una encuesta semiestructurada dirigida a los jefes de la unidad de producción del cultivo de maíz, donde se incluyeron preguntas relacionadas con datos generales del productor, producción, aspectos socioeconómicos y ambientales. En cuanto al diseño de la investigación, se efectuó mediante la implementación del marco para MESMIS, ya que es un modelo metodológico o matriz de referencia dinámico no estático, que evoluciona de acuerdo con las necesidades inmediatas para enriquecer el desempeño de este y es acorde al objeto de estudio de análisis por su escala de evaluación y especificidad del mismo (Astier et al., 2002).

En este sentido, el marco plantea seis pasos generales que surgen de la intersección de procesos ambientales con factores sociales y económicos. Por lo tanto, para alcanzar los objetivos esperados, se aplicaron: definición del sistema, determinación de puntos críticos del sistema, criterios de diagnóstico y uso de indicadores económicos, sociales y ambientales, medición y monitoreo de indicadores, presentación e integración de resultados, así como conclusiones y recomendaciones.

Derivado de lo anterior, una vez definido el sistema a evaluar como parte del diagnóstico del sistema de producción de maíz, tal y como sugieren Masera et al. (2000) con el objeto de identificar los puntos vulnerables que incidieron sobre el agroecosistema de maíz con relación en la sustentabilidad, se distinguieron los puntos críticos sobre el sistema productivo. Posteriormente, se determinaron los criterios de diagnóstico e indicadores para la medición de sustentabilidad. Para la selección de indicadores, se relacionaron aspectos del ámbito económico, sociales y ambientales, de evaluación y monitoreo en las unidades de producción participantes de acuerdo con la información obtenida en campo; siendo estos 21 criterios y 23 indicadores (Cuadro 1).

Cuadro 1. Criterios de diagnóstico e indicadores para medir los niveles de sustentabilidad en la producción de maíz en los municipios de Villaflores y La Trinitaria, Chiapas.

\begin{tabular}{|c|c|c|c|c|}
\hline Ámbito & Categoría & Criterios & Indicadores & $\begin{array}{l}\text { Parámetros de } \\
\text { evaluación para el } \\
\text { óptimo }\end{array}$ \\
\hline \multirow[t]{3}{*}{ Económico } & $\begin{array}{l}\text { Benéfico } \\
\text { económico }\end{array}$ & Productividad & Rendimientos & $\begin{array}{l}\mathrm{R}=4 \mathrm{t} \mathrm{ha}^{-1} \\
\text { Villaflores } \mathrm{R}= \\
1.84 \mathrm{t} \mathrm{ha}^{-1} \mathrm{La} \\
\text { Trinitaria }\end{array}$ \\
\hline & & Ingresos & $\begin{array}{l}\text { Costos de } \\
\text { producción } \\
\text { Ingresos netos }\end{array}$ & $\begin{array}{l}\mathrm{CP}<\mathrm{IT}-\mathrm{IN} \\
\mathrm{IN}>\mathrm{IT}-\mathrm{CP}\end{array}$ \\
\hline & & & $\begin{array}{l}\text { Fuente de ingresos } \\
\text { provenientes de otra } \\
\text { actividad no } \\
\text { agrícola }\end{array}$ & $\begin{array}{l}\text { Número de } \\
\text { actividades } \\
\text { económicas }\end{array}$ \\
\hline
\end{tabular}




\begin{tabular}{|c|c|c|c|c|}
\hline Ámbito & Categoría & Criterios & Indicadores & $\begin{array}{l}\text { Parámetros de } \\
\text { evaluación para el } \\
\text { óptimo }\end{array}$ \\
\hline & $\begin{array}{l}\text { Eficiencia } \\
\text { económica }\end{array}$ & $\begin{array}{l}\text { Ganancias } \\
\text { netas }\end{array}$ & Relación B/C & $>1$ \\
\hline & Autosuficiencia & $\begin{array}{l}\text { Físico- } \\
\text { económica }\end{array}$ & $\begin{array}{l}\text { Independencia de } \\
\text { insumos externos }\end{array}$ & $\begin{array}{l}\text { Procedencia } \\
\text { externa mínima } \\
\text { de los materiales } \\
\text { y servicios } \\
\text { utilizados en el } \\
\text { proceso de } \\
\text { producción }\end{array}$ \\
\hline & & $\begin{array}{l}\text { Dependencia } \\
\text { de apoyo } \\
\text { gubernamental }\end{array}$ & $\begin{array}{l}\text { Grado de } \\
\text { dependencia de } \\
\text { apoyos } \\
\text { gubernamentales }\end{array}$ & Nulo apoyo \\
\hline & & Crédito & Acceso a crédito & Nulo acceso \\
\hline \multirow[t]{7}{*}{ Social } & Participación & $\begin{array}{l}\text { Generación de } \\
\text { empleos }\end{array}$ & $\begin{array}{l}\text { Mano de obra } \\
\text { empleada }\end{array}$ & $\begin{array}{l}\text { Mínima } \\
\text { contratación de } \\
\text { jornales ha }\end{array}$ \\
\hline & & $\begin{array}{l}\text { Grado de } \\
\text { participación } \\
\text { de las mujeres } \\
\text { en la UPF }\end{array}$ & $\begin{array}{l}\text { Participación de las } \\
\text { mujeres en } \\
\text { actividades } \\
\text { agrícolas }\end{array}$ & $>1$ \\
\hline & $\begin{array}{l}\text { Cambio e } \\
\text { Innovación }\end{array}$ & Escolaridad & $\begin{array}{l}\text { Nivel de } \\
\text { escolaridad en el } \\
\text { municipio }\end{array}$ & Alto nivel \\
\hline & & $\begin{array}{l}\text { Capacidad y } \\
\text { generación de } \\
\text { conocimientos }\end{array}$ & $\begin{array}{l}\text { Recuperación de } \\
\text { técnicas } \\
\text { tradicionales/ } \\
\text { Asistencia técnica }\end{array}$ & $>0$ \\
\hline & & $\begin{array}{l}\text { Desarrollo y } \\
\text { aplicación de } \\
\text { tecnología }\end{array}$ & $\begin{array}{l}\text { Incorporación de } \\
\text { maquinaria y } \\
\text { equipo; así como } \\
\text { adopción de } \\
\text { prácticas }\end{array}$ & $\begin{array}{l}\text { (\%) de adopción } \\
\text { de adopción de } \\
\text { prácticas }\end{array}$ \\
\hline & $\begin{array}{l}\text { Organización/ } \\
\text { control }\end{array}$ & $\begin{array}{l}\text { Nivel de } \\
\text { gestión }\end{array}$ & $\begin{array}{l}\text { Quien toma de } \\
\text { decisiones } \\
\text { productivas y } \\
\text { comerciales }\end{array}$ & $\begin{array}{l}\text { Igualdad en la } \\
\text { organización } \\
\text { social }\end{array}$ \\
\hline & & $\begin{array}{l}\text { Formación a } \\
\text { organización o } \\
\text { asociación } \\
\text { productiva }\end{array}$ & $\begin{array}{l}\text { Pertenencia a } \\
\text { alguna asociación u } \\
\text { organización }\end{array}$ & 10 \\
\hline
\end{tabular}




\begin{tabular}{|c|c|c|c|c|}
\hline Ámbito & Categoría & Criterios & Indicadores & $\begin{array}{l}\text { Parámetros de } \\
\text { evaluación para el } \\
\text { óptimo }\end{array}$ \\
\hline \multirow[t]{8}{*}{ Ambiental } & $\begin{array}{l}\text { Diversificación } \\
\text { productiva }\end{array}$ & $\begin{array}{l}\text { Producción } \\
\text { agrícola y } \\
\text { pecuaria }\end{array}$ & $\begin{array}{l}\text { Número de especies } \\
\text { y razas cultivadas y } \\
\text { manejadas }\end{array}$ & $>1$ \\
\hline & $\begin{array}{l}\text { Vulnerabilidad } \\
\text { Biológica }\end{array}$ & $\begin{array}{l}\text { Existencia de } \\
\text { plagas y } \\
\text { enfermedades }\end{array}$ & $\begin{array}{l}\text { Tipo de plagas y } \\
\text { enfermedades }\end{array}$ & Nula incidencia \\
\hline & & $\begin{array}{l}\text { Eventos } \\
\text { climatológicos }\end{array}$ & $\begin{array}{l}\text { Presencia de } \\
\text { eventos } \\
\text { climatológicos }\end{array}$ & Nula presencia \\
\hline & $\begin{array}{l}\text { Conservación y } \\
\text { protección de los } \\
\text { recursos naturales }\end{array}$ & $\begin{array}{l}\text { Biodiversidad } \\
\text { vegetal }\end{array}$ & $\begin{array}{l}\text { Abundancia o } \\
\text { existencia de otras } \\
\text { especies vegetales } \\
\text { no agrícolas }\end{array}$ & 10 \\
\hline & & $\begin{array}{l}\text { Balance de } \\
\text { materia } \\
\text { orgánica }\end{array}$ & $\begin{array}{l}\text { Incorporación de } \\
\text { MO }\end{array}$ & Sí incorpora \\
\hline & & $\begin{array}{l}\text { Control } \\
\text { químico }\end{array}$ & $\begin{array}{l}\text { Uso pesticidas y } \\
\text { fertilizantes }\end{array}$ & Nulo uso \\
\hline & & $\begin{array}{l}\text { Prácticas de } \\
\text { conservación } \\
\text { del suelo }\end{array}$ & $\begin{array}{l}\text { Incorporación de } \\
\text { prácticas de } \\
\text { conservación y } \\
\text { manejo de suelo }\end{array}$ & Alta presencia \\
\hline & & $\begin{array}{l}\text { Externalidades } \\
\text { generadas }\end{array}$ & $\begin{array}{l}\text { Grado deterioro del } \\
\text { recurso natural }\end{array}$ & $\begin{array}{l}\text { Nulo nivel de } \\
\text { deterioro }\end{array}$ \\
\hline
\end{tabular}

Elaborado con base en Astier et al. (1994).

Igualmente, para estimar la fiabilidad de los 23 indicadores, se efectuó a través del coeficiente Alfa de Cronbach. El valor obtenido fue de 0.925 de un máximo de uno; en donde cuanto más cerca se encuentre el valor alfa a uno, mayor consistencia interna de los ítems analizados. Por lo que, de acuerdo con el criterio de Gliem y Gliem (2003), un valor de alfa mayor a 0.8, se consideró excelente o razonable la medición. En lo que respecta a las escalas de medición, se obtuvieron bajo un enfoque mixto. Una vez obtenido los datos de campo, con el procesamiento de la información se determinaron los valores óptimos, así como la ponderación y estandarización.

De esta forma, la estandarización de los indicadores consistió en la transformación de los valores obtenidos en variables adimensionales que coadyuvaron la comparación de estos mediante la construcción de una escala Likert. De acuerdo con Sarandón y Flores (2009), los indicadores son directos: a mayor valor, más sustentable. En este caso, se consideró el rango de 2 a 10, siendo 2 la categoría menos sustentable o inaceptable, 4 limitado, 6 moderado, 8 bueno y 10 lo más sustentable u óptimo. El valor óptimo está determinado bajo el supuesto de que este nivel mejora el sistema o lo mantiene de forma equilibrada. 
Adicionalmente, en cuanto a la ponderación de los indicadores, se empleó la estadística descriptiva mediante promedios ponderados con escalas tipo Likert, donde el cálculo consistió a partir de la frecuencia de cada ítem fue multiplicada por el valor asignado dentro de la escala estandarizada; y sumando cada uno de los productos de los ítems, para después dividir entre el número de la muestra de cada municipio (Levin y Rubin, 2004). Finalmente, se analizaron los resultados mediante la integración gráficos ameba, donde se señalan los valores obtenidos. La comparación de las áreas máximas de cada sistema determina la sostenibilidad global relativa entre ellos (Sepúlveda, 2008).

\section{Resultados y discusión}

\section{Evaluación de los indicadores de la dimensión económica}

El análisis económico se elaboró con base en las condiciones reales de producción espacial y temporal del sistema de producción y calculada por municipios; que se visualiza a continuación en la Figura 1.

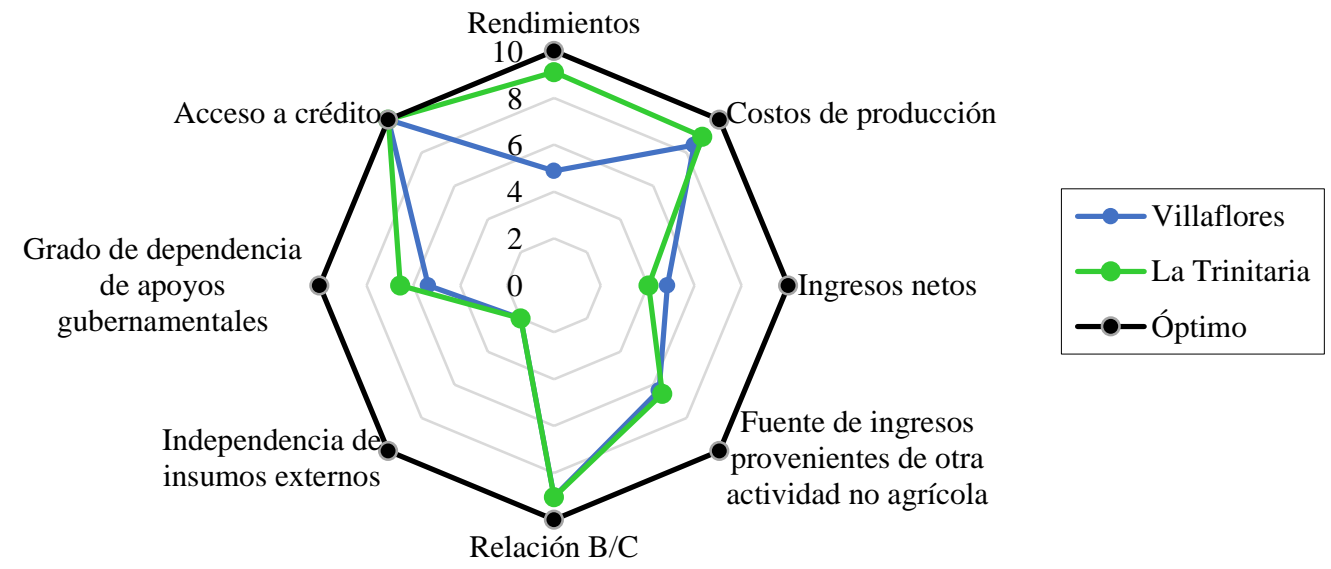

Figura 1. Radial de los indicadores de la dimensión económica.

En primer lugar, el indicador de rendimientos del cultivo de maíz expresa una variabilidad significativa entre los municipios, dada las condiciones agroclimatológicas, la adaptabilidad del sistema productivo en la zona, el tipo de tecnología y semilla empleada, difiriendo en los rendimientos esperados. Es de resaltar, que los altos rendimientos, se deriva de la implementación de tecnología y uso de agroquímicos que potencializan la producción del cultivo.

Sí bien la implementación de tecnología, fertilizantes y pesticidas incide en el aumento de los rendimientos, también incrementa en los costos de producción del maíz por el alto precio de los insumos y de los costos variables como el arrendamiento, mantenimiento y renovación de la maquinaria. Además, los planteamientos de Cruz et al. (2004), manifiestan que los costos de producción son mayores en la tecnología de tractor. De esta forma, se coincide con Sangerman et al. (2009) donde expresa que el uso de tecnologías es un factor esencial para una mayor rentabilidad y se vislumbra una tendencia de incremento en los costos de producción, en la medida que se usa tecnología más desarrollada y decrecientes en las tecnologías menos desarrolladas. 
En cuanto al indicador de ingresos netos por venta de producción, se refleja un ingreso limitado a pesar de una rentabilidad buena de la producción. Por ende, los recursos económicos son insuficientes para el sostenimiento de la familia, de ahí la necesidad de diversificar la economía. De igual forma, Arias (2009) indica que la producción agrícola ha dejado de ser suficiente, por lo que los productores se ven en la necesidad de diversificar sus fuentes de ingresos mediante actividades pluriactivas. Adicionalmente, Carvalho y Moesch (2013) manifiesta que los beneficios de la diversificación de actividades no agrícolas en el medio rural consisten en el aumento de los recursos económicos, que incentiva el desarrollo económico, social y ambiental.

Entonces, tanto los costos de producción como la relación beneficio-costo, cuentan con un nivel de 9.02 para Villaflores y 9.03 para La Trinitaria. Lo que muestra aproximarse al óptimo del indicador. Sin embargo, se requiere generar mayores ingresos de la producción mediante el incremento de la densidad poblacional de siembra y diversificación del mercado de venta de la producción; para lograr que este indicador sea sostenible. Con lo que respecta, a la auto dependencia del sistema, se muestra que es baja, debido al alto uso de insumos externos. Según Astier et al. (2003), indicó en investigaciones, resultados con dependencia del exterior entre 55 y $70 \%$ aproximadamente en los sistemas que contemplaron maquinaria, equipo y agroquímicos.

En lo relativo a la intervención de instituciones gubernamentales en otorgamiento de apoyos o subvenciones, existe un limitado y moderado grado de dependencia por los apoyos gubernamentales recibidos, siendo un nivel de 5.37 y 6.55 para Villaflores y La Trinitaria, respectivamente. De tal manera, que existen una minoría de productores que reciben apoyo federal de Procampo, Programa de fomento a la agricultura y estatal como el programa Amanecer; destinados a la producción y al mejoramiento de la calidad de vida de la población adulta en situación de pobreza, vulnerabilidad y exclusión social.

En este tenor, de acuerdo con Reyes et al. (2003), se evidencia un efecto negativo de los subsidios sobre la producción, debido que los programas de apoyo generan un sustento económico dependiente, provocando un comportamiento negativo e insostenible en las unidades de producción. En este sentido, tal y como señala García (2017), el no acceder a créditos favorece a los productores a no descapitalizarse y disminuir su producción por la falta de liquidez. Lo anterior, se coincide con el autor dado que la contratación de crédito trae consigo un pago por el financiamiento a tasas altos y en ocasiones no preferenciales, lo que contribuye a no ser rentable y sostenible la producción de maíz. Por lo anterior, se comparte la visión de González et al. (2018), el cual expresan que es indispensable que la agricultura sea más competitiva, impulsada a través de la integración de pequeños productores de maíz en cadenas de valor, vinculados a mercados más redituable e innovación en el uso de tecnología óptima para la producción.

\section{Evaluación de los indicadores de la dimensión social}

Con base a la información de la Figura 2, se tienen siete indicadores que determinaron el nivel de sustentabilidad de esta dimensión. De acuerdo con lo observado, algunos productores de Villaflores contratan mano de obra por jornales que en comparación con los productores de la Trinitaria que recurren a la participación e integración de trabajo familiar en los procesos de producción. Al respecto, como señala Manzano (2009), el contratar personal para este tipo de agricultura, implica un costo significativo para el productor y a la vez, provoca un proceso de intensificación y modernización de las explotaciones agrícolas, con carácter empresarial y con menor motivación por el trabajo que el propio agricultor. 


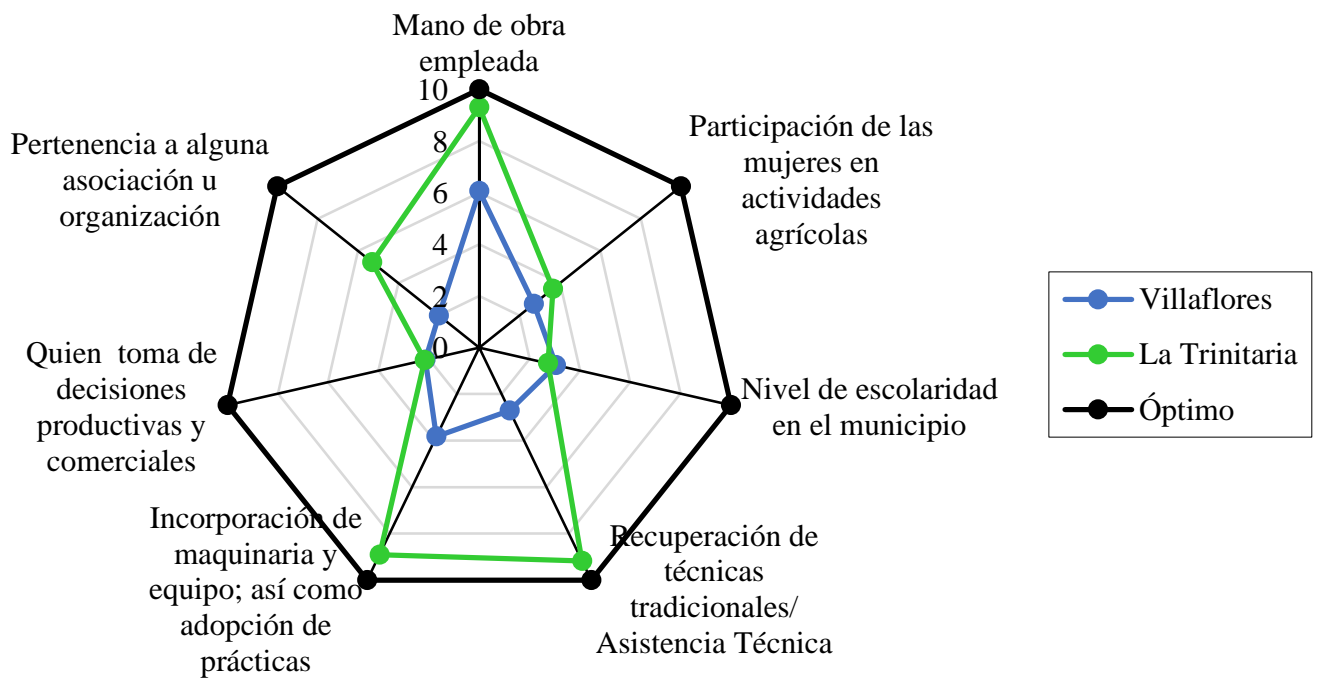

\section{Figura 2. Radial de los indicadores de la dimensión social.}

En lo relativo a la participación de mujeres en actividades agrícolas y toma de decisiones productivas y comerciales, se compara en ambos municipios una limitada participación equitativa en el sistema productivo, así como baja distribución del poder en la toma de decisiones de la actividad agrícola. En este sentido, como infiere Vieyra (2004) el trabajo o participación de las mujeres es aún marcado en la sociedad como un ente pasivo, y poco se habla de los satisfactores que obtienen las mujeres dentro de los medios de producción, como un contribuyente activa y participe de las decisiones en el sistema de producción.

De manera análoga, el grado de escolaridad de los productores, se muestra una variabilidad en este indicador, obteniendo niveles de 3.05 y 2.72 cercanos al rango de inaceptable para la sostenibilidad social. Como lo demuestra Bernal (2014), destaca que la educación favorece la autonomía de las personas y brinda posibilidad de autogestionar sus vidas, mediante la generación de capacidades nuevas y enriquecimiento personal y social; que permite establecer nuevas oportunidades de bienestar y calidad de vida.

Por otra parte, en La Trinitaria aplican técnicas tradicionales y maquinaria manual (animales de trabajo, herramientas como esqueque, coa hoz, azadón, guadaña, etc.), que en comparación con Villaflores que se desarrolla una práctica tecnificada (niveladoras, sembradoras, tractor, etc.). De ahí que, Cruz et al. (2004) plantean la necesidad de usar tecnologías de menor uso de capital y de menor intensidad, pero con mayor desarrollo, porque resultan con un mayor rendimiento utilizando condiciones restrictivas y conservar los recursos naturales.

Para terminar esta dimensión, el indicador pertenencia a alguna asociación u organización de acuerdo con los resultados para los dos municipios, para Villaflores muestra un valor menor al deseable. En contraste, algunos productores de La Trinitaria indican ser miembros afiliados a sociedades de producción rural y a organizaciones sólidas como Organización Campesina Emiliano Zapata, Organización Tziscao Cinco Lagos y Frente Campesino Popular; en el cual, participan en asambleas de socios para la toma de decisiones, obtener beneficios como asegurar la venta de maíz, asignación de apoyos gubernamentales y descuento en insumos. 
Asimismo, es clara muestra con la visión de García (2000), donde manifiesta que la organización de los productores agrícolas constituye una fuerza motora y alternativa para impulsar mejoras sustantivas en la actividad agrícola, al mismo tiempo que conforman unos de los instrumentos fundamentales para el logro de los cambios estructurales requeridos en el proceso de trasformación social del campo y una forma determinante de incorporar al campesino al proceso de desarrollo social y económico del país.

\section{Evaluación de los indicadores de la dimensión ambiental}

Referente a la evaluación de los indicadores ambientales, se consideraron ocho indicadores que construyeron el nivel de sustentabilidad de esta dimensión, como se vislumbra en la Figura 3.

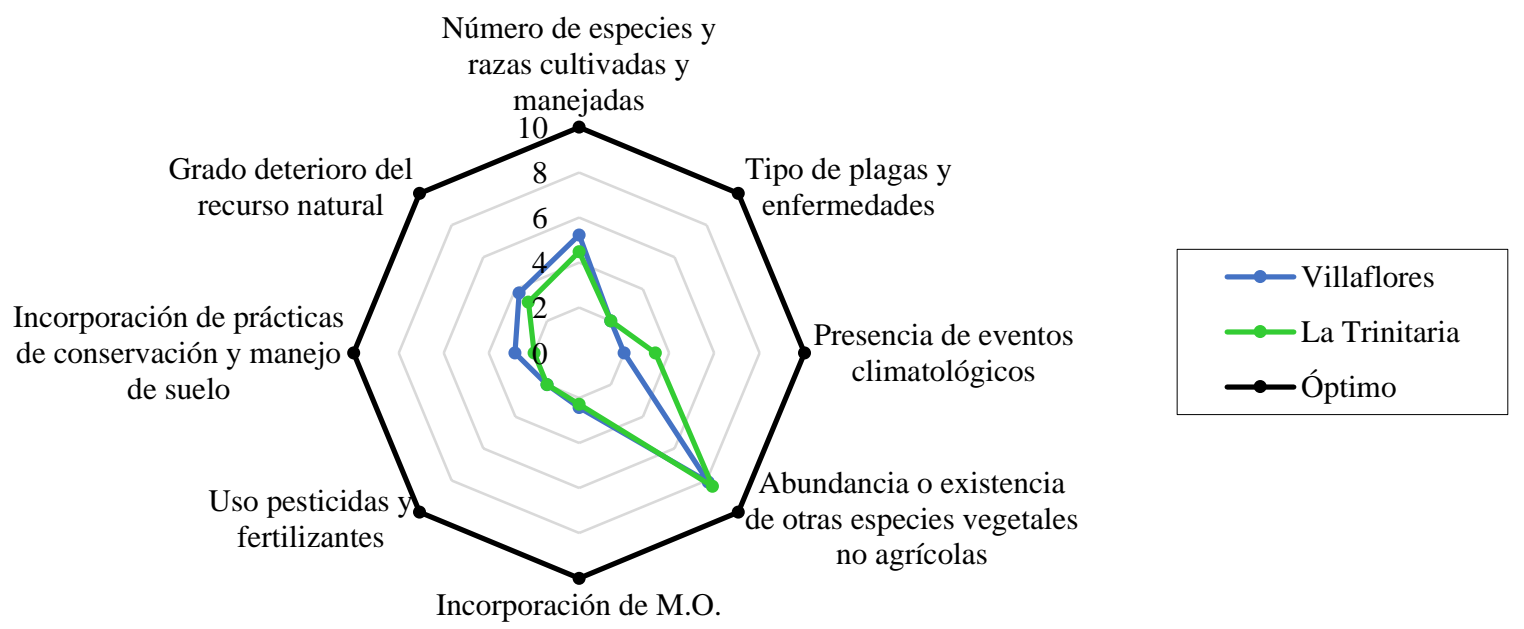

\section{Figura 3. Radial de los indicadores de la dimensión ambiental.}

Como resultado del estudio ambiental, en cuanto a los indicadores de número de especies y razas cultivadas y manejadas, así como abundancia o existencia de otras especies vegetales no agrícolas, en el primer caso se observa que tanto en Villaflores como en La Trinitaria, la mayoría de los productores de maíz cuentan con un valor limitado, dado que en su mayoría de dedican al monocultivo de maíz con poca diversificación productiva agropecuaria.

De acuerdo con Griffon (2008), refieren que el monocultivo es inherente inestable, por lo que se debe considerar la riqueza de los elementos en el sistema, además de tomar en cuenta el número de relacione ecológicas entre los componentes de la agrobiodiversidad. Para el segundo caso, en los dos municipios se refleja un nivel bueno de especies distintas vegetativas a las agrícolas presentes en el entorno; lo que muestra una protección alta del agroecosistema de maíz. En efecto, tal y como sustentan donde la biodiversidad de especies es una estrategia que garantiza la sostenibilidad de los agroecosistemas.

Por otra parte, con relación en la presencia de plagas y enfermedades que exhibe el cultivo de maíz en los distintos municipios, destacan el tizón, chamusco, gusano cogollero, gusano trozador, gusano elotero, y chicharrita de maíz. Consecuentemente, en la mayoría de los productores recurre al uso de agroquímicos como urgá, finale, fipol, sulfato de amonio y herbipol, para controlar las densidades poblacionales de fitófagos y fitopatógenos en la agricultura, aunado al uso de sustancias 
químicas como fertilizantes ante la necesidad de una producción intensiva. El uso de estos productos provoca contaminación del suelo y agua y alteración al medio ambiente, propiciando ser un sistema el sistema productivo de maíz vulnerable biológicamente e insostible.

De esta manera, queda en evidencia que el sistema del monocultivo requiere optar por métodos biológicos como los abonos orgánicos y materia orgánica, que ocasionen menor impacto económico y productivo y que los sistemas sean menos dependientes de agroquímicos, para mantener un balance ecológico de la biodiversidad y efecto positivo en la conservación del suelo y los recursos naturales.

La conservación de suelos evita a perdida de nutrientes fundamentales para mejorar las capacidades productivas de los suelos agrícolas (Damián et al., 2010). También, la incidencia de eventos climatológicos y las alteraciones de los ciclos y procesos naturales perturban el equilibrio del sistema que conforma el medio natural; ocasionando daños económicos directos y caída de la productividad.

\section{Visión general de la evaluación de sustentabilidad del cultivo de maíz}

Derivado de la medición de los indicadores que miden la sustentabilidad en los tres ámbitos el en sistema de producción de maíz para los municipios de Villaflores y La Trinitaria, Chiapas, se exhibe a continuación la gráfica radial que muestra la visión general de la evaluación para determinar el perfil de sustentabilidad, obtenido del planteamiento de los valores óptimo pasa cada indicador, considerando el estado ideal de la misma (Figura 4).

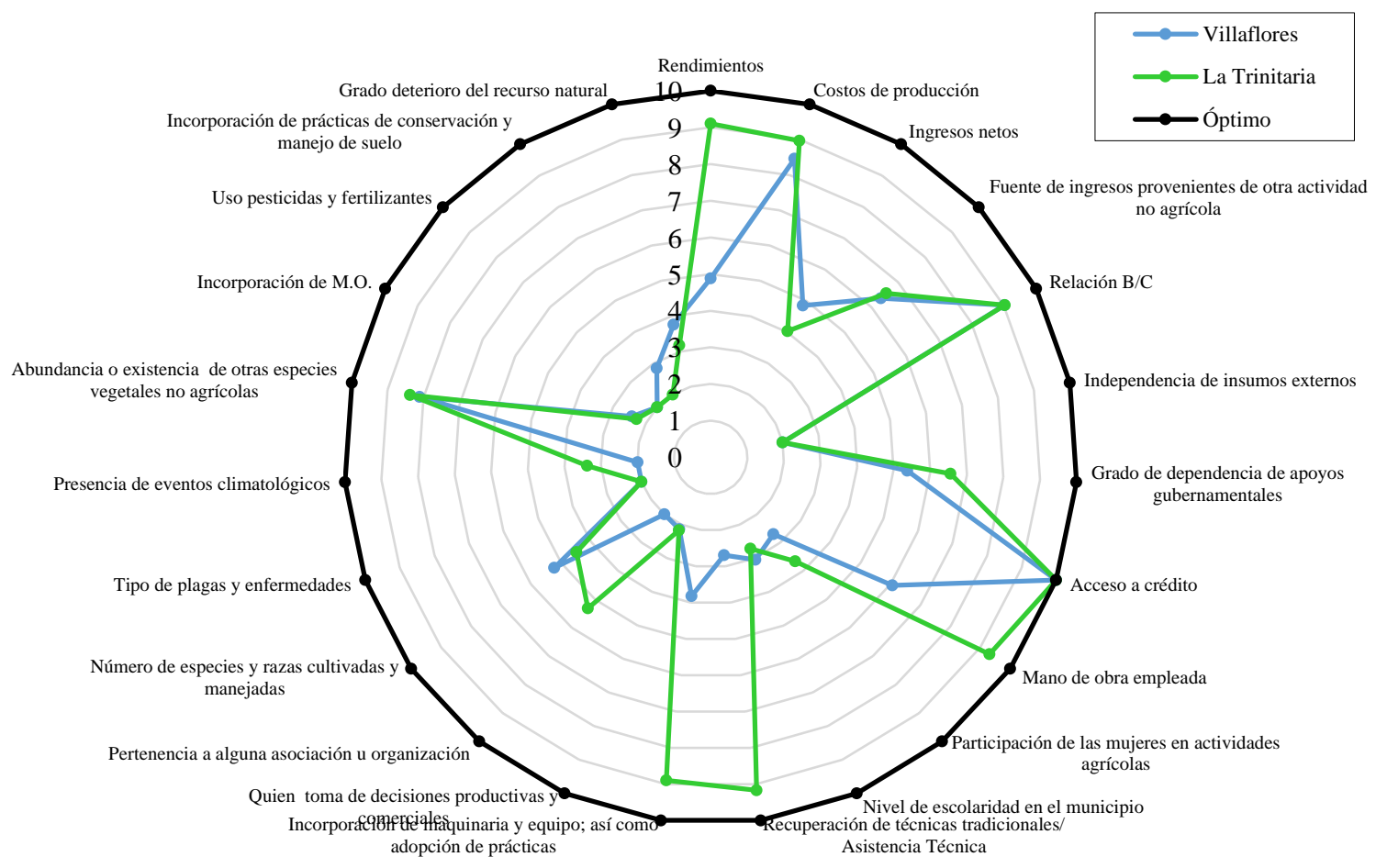

Figura 4. Diagrama tipo AMEBA: integración de los indicadores de sustentabilidad. 
De acuerdo con los resultados obtenidos en la evaluación para Villaflores y La Trinitaria, destaca que en la dimensión económica se encuentra en un nivel sustentable moderado, con un puntaje de 5.77 y 6.32, respectivamente. En este ámbito, se evidencia a partir de los valores obtenidos, aspectos frágiles como ingresos netos por producción, fuente de ingresos por otras actividades no agrícolas, dependencia de insumos externos y grado de dependencia de apoyos gubernamentales.

Con respecto a los indicadores sociales, se refleja valores menores de 3.01 y 5.08 al promedio moderado para los dos municipios. Para el caso de Villaflores, se nota un nivel sustentable vulnerable en los indicadores de mano de obra empleada, recuperación de técnicas tradicionales y asistencia técnica, incorporación de maquinaria y equipo, así como adopción de prácticas, y pertenencia de los productores a alguna asociación u organización.

En cuanto la dimensión ambiental, destaca un nivel sustentable endeble para Villaflores y La Trinitaria, con una puntuación de 3.11 y 3.6 respecto al óptimo, en función a los valores obtenido en todos sus indicadores de esta dimensión, como lo son: especies y razas cultivadas y mejoradas (agrodiversidad) en el cultivo de maíz, tipo de plagas y enfermedades, uso de pesticidas y fertilizantes, presencia de eventos climatológicos, incorporación de materia orgánica, incorporación de prácticas de conservación y manejo de suelo y grado de deterioro del recurso natural. Por lo que no se cumple de forma óptima y agroecológica dada la presencia de factores antropogénicos y ambientales.

Finalmente, es de observarse que los valores de sustentabilidad de los polígonos para los municipios de Villaflores y La Trinitaria, son de 3.78 y 4.81, respectivamente. Por lo tanto, es de notarse que predomina un nivel sustentable limitado en el ámbito económico, social y ambiental, lo que demuestra que, la producción de maíz en estos municipios se aleja del óptimo de la sustentabilidad, como resultado de una baja integración entre las dimensiones.

\section{Conclusiones}

A partir de los resultados significativos que destaca la medición, muestra nivel bajo de sustentabilidad en el agroecosistema. Sin embargo, en el ámbito económico el sistema productivo reflejó un nivel sustentable fortalecido en las categorías de beneficio económico y eficiencia económica, pero vulnerable y frágil en la dimensión social y ambiental, por bajos niveles de sustentabilidad en la participación social equitativa, cambio e innovación, organización social, vulnerabilidad biológica y conservación y protección de los recursos naturales.

Por ende, la evaluación de sustentabilidad en el cultivo de maíz para los municipios de Villaflores y La trinitaria, Chiapas, se vislumbra que el sistema es afectado por distintas presiones sociales, económicas y ambientales que provocan y aceleran cambios en su estructura socioeconómica y ambiental; incidiendo en un escenario de alta fragilidad, situación que hace vulnerable al cultivo de maíz ante las alteraciones que presenta en el sistema de producción.

Además, para mitigar el impacto de la agricultura en el ambiente, se requiere de la aplicación de técnicas agroecológicas, que desarrollen una explotación amigable para el ambiente y donde el proceso de producción del maíz sea estable y no ser opuesto al desarrollo sustentable de la producción agrícola. 


\section{Literatura citada}

Altieri, M. 1994. Biodiversity and pest management un agroecosystems. The Haworth Press. New York. 236 p.

Arias, P. 2009. La pluriactividad rural a debate. In: la pluriactividad en el campo latinoamericano. Carton, G. H. y Martínez, V. L. (Comps.). $1^{\text {ra. }}$ Ed. FLACSO. Ecuador. 309 p.

Astier, M.; López, R. S.; Pérez, A. E. y Masera, O. R. 2002. El marco de evaluación de sistemas de manejo incorporando indicadores de sustentabilidad (MESMIS) y su aplicación en un sistema agrícola campesino en la región Purhepecha, México. In: agroecología: el camino hacia una agricultura sustentable. Sarandón, S. J. (Ed.) Ediciones Científicas Americanas. Buenos Aires, Argentina. 415-430 pp.

Astier, M.; Masera, O.; López, R. S.; Galván, M. Y.; Ortiz, A. T.; García, B. L. E.; García, B. R.; González, C. y Speelman, E. 2008. El Proyecto de evaluación de sustentabilidad MESMIS. In: evaluación de sustentabilidad: un enfoque dinámico y multidimensional. Masera, O. R.;

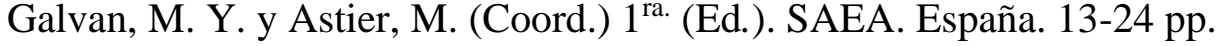

Astier, M.; Pérez, A. E.; Ortiz, T. y Mota, F. 2003. Sustentabilidad de sistemas campesinos de maíz después de cinco años: el segundo ciclo de evaluación MESMIS. LEISA. 19(1):39-46.

Bassols, B. A. 1984. Recursos naturales de México. Nuestro tiempo. México, DF. 299 p.

Bernal, A. 2014. La función de la educación para la creación de las capacidades centrales. Rev. Edetania. 46:123-140.

Carvalho, M. S. and Moesch, M. M. 2013. Turismo como fenômeno social e suas implicações no espaço rural. Rev. Brasileira de Ecoturismo. 6 (2):442-457.

Cruz, L. A.; Martínez, S. T. y Omaña, S. J. M. 2004. Fuentes de fuerza, diversidad tecnológica y rentabilidad de la producción de maíz. Ciencias Naturales y Agropecuarias. 3(11):275-283.

Damián, H. M. A.; Ramírez, V. B.; Aragón, G. A.; Huerta, L. M.; Sangerman, J. D. M. J. y Romero, A. O. 2010. Manejo del maíz en el estado de Tlaxcala, México: entre lo convencional y lo agroecológico. Rev. Latinoam. Rec. Nat. 6 (2):67-76.

Edwards, C. A.; Lai, R.; Madden, P.; Miller, R. H. and House, G. 1990. Sustainable agricultural systems. soil and water conservation society. Iowa, USA. 696 p.

García, L. 2000. Las organizaciones de productores agrícolas en el Marco del proceso de Globalización Económica. Fermentum. 29(10):477-490.

García, N. C. 2017. Las políticas de crédito al sector agropecuario en Nicaragua 1990-2012. Rev. Científ. Electr. Cienc. Hum. 36(12):24-44.

Gliem, J. A. y Gliem, R. R. 2003. Cálculo, interpretación y reporte del coeficiente de confiabilidad alfa de Cronbach para escalas tipo Likert. Revista Publicando. 2(1):62-67.

González, F. S.; Guajardo, H. L. G.; Almeraya, Q. S. X.; Pérez, H. L. M. y Sangerman, J. D. M. 2018. Tipología de productores de maíz en los municipios de Villaflores y La Trinitaria, Chiapas. Rev. Mex. Cienc. Agríc. 9(8):1763-1776.

Griffon, B. D. 2008. Estimación de la biodiversidad en agroecología. Agroecología. 3:25-31.

Levin, R. I. y Rubin, D. S. 2004. Estadística para administración y economía. Séptima edición. Pearson Educación. México. 952 p.

Manzano, A. F. y García, C. A. 2009. Técnicas de estudio de tiempos para la planificación de la mano de obra en el cultivo de tomate de invernadero. Agrociencia. 43(3):267-277.

Masera, O. R.; Astier, M. E. y López, R. S. 2000. Sustentabilidad y manejo de recursos naturales: el marco de evaluación MESMIS. GIRA. Pátzcuaro, Michoacán. 107 p. 
ONU. 1992. Organización de las Naciones Unidas. Agenda 21. Conferencia de las Naciones Unidas sobre el Medio Ambiente y el Desarrollo. ONU. Rio de Janeiro.

ONUAA. 1999. Organización de las Naciones Unidas para la Alimentación y la Agricultura. FAO Focus: la mujer y la seguridad alimentaria. ONUAA.

Reyes, H. H.; Cortina, V. S.; Perales, R. H.; Kauffer, M. E. y Pat, F. J. M. 2003. Efecto de los subsidios agropecuarios y apoyos gubernamentales sobre la deforestación durante el periodo 1990-2000 en la región de Calakmul, Campeche, México. Investigaciones Geográficas. 51:88-106.

Sangerman, J. D. M. J.; Espitia, R. E.; Villaseñor, H. E.; Ramírez, V. B. y Alberti, M. P. 2009. Estudio de caso del impacto de la trasferencia de tecnología en trigo del INIFAP. Agric. Téc. Méx. 1(35):25-37.

Sarandón, S. y Flores, C. 2009. Evaluación de la sustentabilidad en agroecosistemas: una propuesta metodológica. Agroecología. 4:19-28.

Sepúlveda, S. S. 2008. Biograma: metodología para estimar el nivel de desarrollo sostenible en espacios territoriales. Instituto Interamericano de Cooperación para la Agricultura (IICA). San José, Costa Rica. 118 p.

Torres, L. P.; Cruz, C. J.; Acosta, B. R.; Dávila, F. D.; Rodríguez, S. L. y Sánchez, O. J. 2000. Políticas de sustentabilidad ambiental y microempresas agropecuarias cercanas a polos urbanos en expansión. Tendencias del desarrollo regional en México. Universidad Autónoma Metropolitana (UAM). 26 p.

Vieyra, J.; Castillo, A.; Losada, H.; Cortés, J.; Alonso, G.; Ruiz, T.; Hernández, P.; Zamudio, A. y Acevedo, A. 2004. La participación de la mujer en la producción de traspatio y sus beneficios tangibles e intangibles. Cuadernos de Desarrollo Rural. 53:9-23.

Vivanco, A. M. 2005. Muestreo estadístico: diseño y aplicaciones. Universitaria, SA. España. 209 p. 